\title{
Behandlung und Betreuung von zerebral schwerstgeschädigten Langzeitpatienten
}

\author{
Medizinisch-ethische Richtlinien der Schweizerischen Akademie der Medizinischen Wissenschaften*
}

* Mitglieder der für die Ausarbeitung dieser Richtlinien tätigen Subkommission Prof. Hannes Stähelin, Basel, Vorsitz;

Prof. Alberto Bondolfi, Zürich; Prof. Johannes Fischer, Zürich; Prof. Andreas U. Gerber, Biel; Prof. Annemarie Kesselring, Basel; PD Dr. Christian Kind, St. Gallen; Dr. Cornelia Klauser, Agno; Prof. Rudolf Ritz, Binningen; lic. iur. Michelle Salathé, Basel (ex officio);

Dr. Noémi de Stoutz, Ayent; Prof. Günter Stratenwerth, Basel; Prof. Michel Vallotton, Genf, Präs. ZEK;

PD Dr. Gilbert Zulian, Collonge

\section{Vorwort}

Die Zentrale Ethikkommission (ZEK) hat vor einiger Zeit beschlossen, die medizinischethischen Richtlinien zur Begleitung und Betreuung sterbender und zerebral schwerstgeschädigter Patienten aus dem Jahre 1995 zu überarbeiten, da diese der aktuellen Situation nicht mehr entsprechen. Gleichzeitig hat die ZEK entschieden, die Richtlinien aufzuteilen und zwei Subkommissionen mit der Überarbeitung $\mathrm{zu}$ beauftragen. Die eine - unter der Leitung von Dr. Markus ZimmermannAcklin - arbeitet an den Richtlinien zur Begleitung von Sterbenden, die andere - unter der Leitung von Prof. Hannes Stähelin - hat die vorliegenden Richtlinien über die Behandlung und Betreuung zerebral schwerstgeschädigter Patienten verfasst. Diese Gruppe von Patienten können tatsächlich nicht als Sterbende bezeichnet werden, da ihr Zustand über eine sehr lange Zeit unverändert bleiben kann. Der irreversible Verlust des Bewusstseins und der Kommunikationsfähigkeit nimmt ihnen jedoch jede Möglichkeit der Selbstbestimmung. Zusätzlich macht sie dieser Zustand (mit all seinen körperlichen Folgeerscheinungen) extrem abhängig und verletzlich. Daraus ergibt sich eine ganze Reihe von Problemen für das betreuende Team - Ärzte und Pflegende - in bezug auf Entscheide hinsichtlich therapeutischer Massnahmen (z.B. lebensverlängernde
Behandlung, palliative Pflege, künstliche Flüssigkeits- und Nahrungszufuhr), und auf Entscheide am Lebensende. In jedem Fall muss das Pflegeteam klare Therapieziele festlegen.

Der Akzent liegt auf dem Anspruch dieser Personen auf Autonomie - auch wenn sie diese nicht mehr ausüben können -, auf ihren Rechten sowie auf der Ermittlung ihres Willens (dem mutmasslichen oder demjenigen, wie er in einer Patientenverfügung festgehalten ist).

In den Richtlinien wird auch das Problem der ärztlich-pflegerischen Rahmenbedingungen angesprochen; die neuen Richtlinien sollen dazu beitragen, dass Entscheide in Einzelfällen nicht durch ökonomische Überlegungen beeinflusst werden.

Gerne sprechen wir dem Präsidenten und allen Mitgliedern der Subkommission unseren besten Dank für ihr Engagement und ihre wertvolle Arbeit aus.

Der Entwurf für diese Richtlinien geht nun in die Vernehmlassung. Die SAMW erwartet Kritiken, Anregungen und Änderungsvorschläge von seiten der interessierten Kreise. Diese können bis zum 31. März 2003 an das Generalsekretariat der SAMW gesandt werden (Petersplatz 13, 4051 Basel, Fax 06126990 39; E-Mail: mail@samw.ch).

Prof. Michel B. Vallotton, Präsident der ZEK

Prof. Werner Stauffacher, Präsident der SAMW

\footnotetext{
1 Im Interesse der leichteren Lesbarkeit des Textes wird in der Folge durchwegs die männliche Bezeichnung von Personen verwendet. Die entsprechenden Texte betreffen immer auch die weiblichen Angehörigen der genannten Personengruppen.
}

\section{Präambel}

Zerebral schwerstgeschädigte Langzeitpatienten [1] sind Menschen, bei welchen krankheitsoder verletzungsbedingte Hirnschädigungen zu einem langdauernden Zustand persistierender Bewusstlosigkeit oder schwerster Beeinträchtigung des Bewusstseins mit voraussichtlich irreversiblem Verlust der Kommunikationsfähigkeit geführt haben. Ein Wiedererlangen des Bewusstseins oder der selbständigen Willensäusserungen ist kaum mehr zu erwarten.
Bei zerebral schwerstgeschädigten Langzeitpatienten ist die Fähigkeit zur Selbstbestimmung verloren. Der betroffene Patient ist weitgehend fremdbestimmt. Andere müssen für ihn entscheiden; dabei sind jedoch die Persönlichkeitsrechte zu wahren.

Die stellvertretende Wahrnehmung der Interessen eines zerebral schwerstgeschädigten Langzeitpatienten ist heikel und die Entscheidungsprozesse sind komplex. Idealerweise liegt eine aktuelle, genügend detaillierte Patientenverfügung vor. Fehlt diese jedoch, muss der mut- 
massliche Wille des Patienten herangezogen werden. Eine zusätzliche Schwierigkeit ergibt sich aus der Unsicherheit der Prognose. Während bei verletzungsbedingten Hirnschädigungen auch bei einem längerandauernden bewusstlosen $\mathrm{Zu}$ stand mit einer Erholung gerechnet werden darf, ist bei krankheitsverursachten schwersten Hirnschädigungen die Prognose wesentlich ungünstiger, doch kann auch in diesen Fällen keine definitive Aussage gemacht werden.

Aus dieser Unsicherheit der Prognose ergeben sich für das betreuende Team häufig schwierige ethische Fragen, vor allem, wenn der Wille des Patienten unbekannt oder nicht eindeutig ist. Insbesondere bei interkurrent auftretenden Komplikationen stellt sich die Frage nach der Fortsetzung einer bereits eingeleiteten Behandlung und jene nach der Aufnahme neuer therapeutischer Massnahmen. Die vorliegenden Richtlinien sollen bei diesen Entscheiden eine Hilfe sein.

\section{Richtlinien}

\section{Geltungsbereich}

Schwerste Hirnschädigungen mit anhaltendem, nicht behandelbarem Verlust der Kommunikationsfähigkeit lassen sich in drei Hauptgruppen aufteilen:

- Der persistierende vegetative Status (PVS, Wachkoma): Ein komatöser Zustand nach krankheits- oder verletzungsbedingter, meist hypoxischer Hirnschädigung kann in einen «vegetativen Status», einen «Wachzustand ohne fassbare Wahrnehmung», übergehen. Dauert der vegetative Status länger als einen Monat (diagnostischer Begriff mit Blick auf die bisherige Situation), spricht man vom "persistierenden vegetativen Status» (PVS); ist dieser höchstwahrscheinlich irreversibel geworden (prognostischer Blick), spricht man vom «permanenten vegetativen Status».

- Schwerste degenerative Hirnerkrankungen im Spätstadium (z. B. Alzheimer-Krankheit): Diese sind charakterisiert durch schwersten kognitiven Abbau [2] (Wortschatz auf einzelne Worte reduziert, keine verbale Kommunikation mehr möglich, Verlust der motorischen Fertigkeiten, in allen Belangen auf Pflege angewiesen); andere Pathologien können ausgeschlossen werden.

- Schwerste bei Geburt vorliegende oder in der frühen Kindheit erworbene Hirnschädigungen: Das Hirn ist durch einen hypoxischischämischen, traumatischen, infektiösen oder metabolischen Prozess oder durch Fehl- bildung so schwer geschädigt, dass die Erlangung kommunikativer Fähigkeiten und einer minimalen Selbständigkeit nicht erwartet werden kann.

Aus den obigen Diagnosen ergeben sich unterschiedliche Konsequenzen für die Betreuung und die Behandlung. Die vorliegenden Richtlinien berücksichtigen die unterschiedliche Situation dieser Patientengruppen.

\subsection{Beschreibung der Patientengruppen}

1.1.1 Persistierender vegetativer Status (PVS) Unter «vegetativem Status» wird ein totaler Verlust der erkennbaren Wahrnehmungsfähigkeit von sich selbst und von der Umgebung verstanden. Die noch teilweise oder vollständig erhaltenen Funktionen von Hypothalamus und Hirnstamm reichen - zusammen mit medizinischer und pflegerischer Unterstützung - zum Überleben aus. Zeichen von wiederholten, reproduzierbaren, willentlich gerichteten Antworten auf visuelle, auditorische, taktile und schmerzende Stimuli fehlen; ebenso sind keine Zeichen von sprachlichem Verständnis oder Ausdruck vorhanden. Es besteht Darm- und Blaseninkontinenz. Teilweise erhalten sind dagegen die Hirn- (pupillar, oculocephal, corneal, vestibulo-ocular) und Spinalreflexe sowie ein Schlaf-Wach-Rhythmus.

\subsubsection{Neurodegenerative Erkrankungen}

Diese Patienten haben einen weitestgehenden Verlust der kognitiven Fähigkeiten aufgrund einer schweren degenerativen Hirnschädigung erlitten und bleiben über Monate in einem stationären Zustand. Dies kann im wesentlichen in den Spätstadien der Alzheimerkrankheit, der Parkinsonkrankheit, der frontotemporalen Demenzerkrankung, in fortgeschrittener Chorea Huntington und bei vaskulärer Demenz eintreten. In der Regel verlaufen andere neurodegenerative Erkrankungen verhältnismässig rasch progredient und betreffen die Kognition erst in einem terminalen Stadium. Erst wenn die Kommunikationsfähigkeit verlorengegangen und eine erkennbare Wahrnehmung nicht mehr vorhanden ist, kommen die vorliegenden Richtlinien zur Anwendung.

1.1.3 Bei der Geburt vorliegende oder in früher Kindheit erworbene Hirnschädigung

Die Situation bei Kindern weist altersabhängig drei ethisch relevante Besonderheiten auf. Diese sind bei Neugeborenen und Säuglingen besonders stark ausgeprägt: 1 . Die Schädigung des Hirns betrifft ein sich entwickelndes Zentralnervensystem. Dies erschwert einerseits die Ab-
Stages (FAST) von Reisberg et al. Stufe 7. 
schätzung des Ausmasses einer aktuellen funktionellen Schädigung, da bei Neugeborenen und Säuglingen viele Funktionen noch nicht manifestiert werden. Zusätzlich besteht auch ein viel grösseres Spektrum der möglichen funktionellen Erholung infolge der sehr grossen Plastizität des kindlichen Nervensystems. Prognosen sind deshalb immer durch eine erhebliche Unsicherheitsspanne gekennzeichnet. 2. Es gibt keine Hinweise auf einen mutmasslichen Willen bezüglich der medizinischen Behandlung. Bei angeborenen Schädigungen gibt es auch keinerlei biographische Anhaltspunkte für Vermutungen zur subjektiven Einschätzung der Lebensqualität durch den Patienten. 3. Kinder sind biologisch, psychisch, sozial und rechtlich in höchstem Mass von ihren Eltern abhängig. Die Folgen medizinischer Behandlungsentscheide bei ungünstiger Prognose betreffen die Eltern sehr direkt und unter Umständen lebenslänglich.

Diese Besonderheiten verunmöglichen die scharfe definitorische Abgrenzung einer Gruppe von Neugeborenen, Säuglingen und Kleinkindern, für welche die vorliegenden Richtlinien zur Anwendung kommen sollen. Vielmehr sollte für jedes einzelne Kind, bei dem durch eine schwere Hirnschädigung die Entwicklung zur Kommunikationsfähigkeit und zu einer minimalen Selbständigkeit in Frage gestellt ist, aufgrund seiner individuellen Prognose darüber entschieden werden, ob eine Modifikation des Therapiezieles im Sinne dieser Richtlinien angemessen ist. Dabei ist der voraussehbare Gewinn an Lebensfreude, Beziehungsmöglichkeiten und Erlebnisfähigkeit, der einem Kind durch eine volle medizinische Therapie ermöglicht werden kann, gegen die Belastung abzuwägen, die in Form von Schmerzen, Diskomfort und Einschränkung mit dieser Therapie verbunden ist.

\subsection{Abgrenzung zu den Sterbenden}

Zerebral schwerstgeschädigte Langzeitpatienten sind mit Sterbenden nicht gleichzusetzen. Sie befinden sich noch in einem stabilisierten, aber mutmasslich irreversiblen Zustand. Interkurrent auftretende Komplikationen oder der Entscheid über einen Therapieabbruch können den Sterbeprozess jedoch in Gang setzen. Bei den Sterbenden ist die verbleibende Lebensspanne relativ kurz und der terminale Prozess progredient. Die Betreuung von sterbenden Patienten wird in einer separaten Richtlinie [3] behandelt.

\section{Patientenrechte}

\subsection{Grundsatz}

Gegenüber Langzeitpatienten mit schwerster Hirnschädigung besteht die Pflicht, in jeder Weise zu helfen und Leiden zu lindern. Die Pflicht zur Lebenserhaltung unterliegt jedoch Einschränkungen. Vorrangig massgebendes Kriterium für Entscheide, auf lebenserhaltende Massnahmen $\mathrm{zu}$ verzichten oder sie abzubrechen, ist der $\mathrm{Pa}-$ tientenwille.

\subsection{Mutmasslicher Wille}

Geht dieser Wille aus einer Patientenverfügung hervor, so ist er zu befolgen, solange keine konkreten Anhaltspunkte dafür bestehen, dass die Patientenverfügung dem derzeitigen Willen des Patienten nicht mehr entspricht. Patientenverfügungen gelten um so eher, je klarer sie formuliert sind, je kürzer die Unterzeichnung zurückliegt und je besser der Patient die eingetretene Situation antizipiert hat. Fehlt es an einer solchen Verfügung, so muss versucht werden, den mutmasslichen Willen des Patienten zu ermitteln.

Eine wesentliche Rolle spielt dabei, wie er in seinem bisherigen Leben gedacht und gehandelt hat und welches seine Präferenzen waren. Informationen darüber sollten insbesondere von einem vom Patienten ausdrücklich benannten Beauftragten oder von den dem Patienten nächststehenden Personen eingeholt werden.

Bei Unmündigen gilt grundsätzlich der Wille des gesetzlichen Vertreters. Eltern sind bei Entscheidungen über Leben und Tod jedoch oft überfordert. Entscheide über die Behandlung und Betreuung sollen im besten Interesse des Kindes im Konsens mit den Eltern bzw. den gesetzlichen Vertretern getroffen werden. Ist bei lebenswichtigen Entscheiden ein Konsens nicht zu erreichen, so ist die Vormundschaftsbehörde beizuziehen.

\section{Entscheidungsprozesse}

Besondere Aufmerksamkeit verdient der Prozess der Entscheidungsfindung. Grundlage der Entscheidung über Ziele (und Ort) der Behandlung und Betreuung sind der Zustand und die Prognose bezüglich Lebensdauer und -qualität sowie die Persönlichkeit und der mutmassliche Wille des Patienten. Dabei sind in die Entscheidungsprozesse die Erfahrung und Sicht der Angehörigen sowie des Pflegeteams miteinzubeziehen. Die so getroffenen Entscheide sollten von allen beteiligten Personen akzeptiert und möglichst mitverantwortet werden können. Klinische Ethikkommissionen [4] können für die Entscheidungsfindung beigezogen werden. Die 
letzte Entscheidungsverantwortung bleibt beim behandelnden Arzt. Entscheide, welche zum Abbruch von lebenserhaltenden Massnahmen führen, müssen protokolliert werden, so dass sie auch im nachhinein noch nachvollziehbar sind.

\section{Behandlung und Betreuung}

\subsection{Grundsatz}

Die therapeutischen Ziele bestimmen das Vorgehen. Palliativbetreuung und -pflege müssen parallel zu den übrigen therapeutischen Massnahmen eingeleitet und unabhängig davon weitergeführt werden. Der angemessene Einsatz der zur Verfügung stehenden Mittel ist prinzipiell geboten und periodisch zu überprüfen. Die Entscheidverantwortlichen haben in ihrem Bereich Mitverantwortung für die gerechte Verteilung der verfügbaren Ressourcen. Dies darf jedoch nicht über die Unterlassung oder den Abbruch von Massnahmen im Einzelfall entscheiden.

\subsection{Therapeutische Massnahmen}

Die therapeutischen Massnahmen ergeben sich aus dem Behandlungsziel. Es gibt Situationen, in denen sonst angemessene Diagnostik- und Therapieverfahren nicht mehr indiziert sind, sondern Begrenzung geboten sein kann. In speziellen Situationen kann der zeitlich befristete Einsatz von therapeutischen Massnahmen erwogen werden. Eine Änderung des Behandlungsziels kommt insbesondere dann in Betracht, wenn die Krankheit weit fortgeschritten ist, so dass eine lebenserhaltende Behandlung nur Leiden verlängert. Unter diesen Umständen ist der Einfluss der therapeutischen Massnahmen auf die Lebenserhaltung und -qualität zu berücksichtigen. Auf invasive und aufwendige Therapiemassnahmen kann eher verzichtet werden als auf schonende und einfache. Mit dem Zurücknehmen von kurativ-therapeutischen Massnahmen findet eine Verschiebung Richtung Pflege und Begleitung statt.

\subsection{Palliation und Pflege}

Zerebral schwerstgeschädigte Langzeitpatienten haben ein Recht auf adäquate palliative Massnahmen. Es handelt sich dabei weitgehend um präventive, komfortvermittelnde Massnahmen (Medizin, Pflege, Physiotherapie usw.). Da diese Patienten ihre Befindlichkeit nicht mitteilen können, kann nicht auf Beschwerden reagiert werden. Darum muss proaktiv nach Zuständen gesucht werden, die aufgrund der klinischen Erfahrung zu Klagen Anlass geben würden. Mit palliativer Zielsetzung sollen Massnahmen auch dann angewendet werden, wenn dadurch die
Lebensdauer verkürzt wird. Zur Palliation gehören wesentlich auch die Begleitung und Instruktion der dem Patienten nahestehenden Personen.

Zur Pflege gehört das Erhalten der körperlichen Integrität, der Beweglichkeit und des Aussehens des Patienten. Pflege soll so kontinuierlich wie möglich angeboten werden. Dies erleichtert den Kontakt zum Patienten und schafft die Möglichkeit, den Patienten und seine Angehörigen näher kennenzulernen.

\subsection{Flüssigkeit und Nahrung}

Ohne gegenteilige Willensäusserung ist die adäquate Zufuhr von Flüssigkeit und Nahrung (enteral oder parenteral) weiterzuführen. Treten im Zusammenhang damit Komplikationen auf, ist die Situation neu zu prüfen. Die Indikation zur Neuaufnahme einer enteralen Sondenernährung sollte sorgfältig geprüft werden.

Von einer Zufuhr von Flüssigkeit ohne gleichzeitige Nährstoffabgabe ist grundsätzlich abzusehen. In terminalen Situationen kann die alleinige Flüssigkeitszufuhr gerechtfertigt sein oder es kann sogar - im Konsens mit dem Team und den Angehörigen - auf die Flüssigkeitszufuhr verzichtet werden. Bei Neugeborenen darf auf Nahrungs- und Flüssigkeitszufuhr nur verzichtet werden, wenn die Etablierung einer enteralen Ernährung mit grossen, belastenden Eingriffen verbunden oder überhaupt nicht möglich ist.

\section{Kommentar}

\section{Ad Präambel}

Die Richtlinien sollen helfen, für den Patienten im Einzelfall die richtige Entscheidung über die Behandlung und Betreuung zu treffen, ohne diese zu präjudizieren. Sie sollen ausserdem ein Hilfsmittel sein beim Erstellen von internen Leitlinien.

\section{Ad 1. (Geltungsbereich)}

Die Krankheitsbilder sind gekennzeichnet durch den praktisch vollständigen Verlust der Kommunikationsfähigkeit, so dass Bedürfnisse und Wünsche nicht mitgeteilt werden können [5].

Die Langzeitprognose und die Feststellung der Irreversibilität eines «persistierenden vegetativen Status» sind äusserst schwierig; sie sind unter anderem abhängig vom Alter des Patienten, von der bisherigen Dauer des Zustandes, von Begleiterkrankungen sowie insbesondere von der Ursache der ursprünglichen Hirnschädigung. So bleiben Erholungschancen beim PVS nach Schädelhirntrauma länger erhalten, d.h. weit über ein Jahr hinaus, als nach krankheits- 
bedingter Hirnschädigung. Im ersten Fall müssen unterstützende Massnahmen (diagnostische, therapeutische, rehabilitatorische und psychische) mit Geduld über längere Zeit fortgesetzt werden. Fragen eines möglichen Therapieverzichtes/-abbruches und/oder der Verlegung sollen frühzeitig, aber ohne Eile in Betracht gezogen werden.

Ad 2.2 (Patientenrechte: Mutmasslicher Wille) Beim Demenzkranken, der aufgrund seiner Erkrankung die Nahrung nicht mehr adäquat schluckt, ist eine Schluckstörung oder gastrointestinale Pathologie (Ösophagus, Magen, Mund, Rachen) auszuschliessen. Das oft beobachtete Verhalten von Dementen, die Nahrung zu verweigern, ist nach zumutbarer Diagnostik als verbindliche Willensäusserung zu werten.

Im Rahmen der palliativen Massnahmen sollen aber Nahrung und Flüssigkeit immer wieder angeboten werden.

\section{Ad 3. (Entscheidungsprozesse)}

$\mathrm{Zu}$ jeder Entscheidung gehören eine Zieldefinition, die Konsenssuche mit allen Beteiligten, eine regelmässige Überprüfung, ob das Ziel erreicht bzw. noch adäquat ist, und wesentlich auch die Klärung der Konsequenzen des Entscheids für alle Beteiligten (z. B. Behandlung neu auftretender Krankheiten, Verlegung in eine andere Institution, Schwierigkeiten für Angehörige bei Besuchen usw.). Solche Entscheidungsprozesse benötigen die hierfür notwendigen Rahmenbedingungen von Raum und Zeit. Entscheidungsprozesse sollen wenn immer möglich nach einer verbindlichen internen Richtlinie durchgeführt werden.

\section{Ad 4.1 (Behandlung und Betreuung: Grundsatz)}

Angemessenheit bedeutet, unter Abwägung der Vor- und Nachteile für den Patienten und in Berücksichtigung der zur Verfügung stehenden Ressourcen den optimalen Weg zwischen «therapeutischem Übereifer» («acharnement thérapeutique») und «therapeutischem Nihilismus» zu suchen.

\section{Ad 4.3 (Behandlung und Betreuung: Palliation und Pflege)}

Neben der regelmässigen klinischen Untersuchung, der gezielten Suche nach Nebenwirkungen laufender Therapien und der Überwachung vegetativer Parameter, die auf Beschwerden hin- deuten können, sind auch die Beobachtungen und Intuition jener Personen ernst zu nehmen, die viel Zeit beim Patienten verbringen (Angehörige, Pflegende). Der Wert mancher Massnahmen kann am ehesten beurteilt werden, wenn sie versuchsweise eingesetzt werden (ex juvantibus). Im Patientenzimmer soll eine ruhige, empathische Atmosphäre herrschen; Kontakte sollen möglichst erhalten werden; die Akzeptanz der Dauer des vegetativen Zustandes und allenfalls eines Therapieabbruches soll reifen können; Angehörige sollen das Geschehen in ihren eigenen Lebensentwurf integrieren können.

\section{Ad 4.4 (Behandlung und Betreuung: Flüssigkeit und Nahrung)}

Bei Neugeborenen darf, wenn eine enterale Ernährung durch wenig belastende Massnahmen (z.B. nasale Magensonde, perkutane Gastrostomie, operative Korrektur einer Duodenalatresie) möglich ist, angesichts der immer unsicheren Prognose auf die Zufuhr von Nahrung und Flüssigkeit nicht verzichtet werden. Wo hingegen eine enterale Ernährung nur um den Preis grosser, belastender Eingriffe oder überhaupt nicht möglich ist, kann der Verzicht auf jegliche Kalorien- und Flüssigkeitszufuhr unter optimaler Sedation und ständiger menschlicher Nähe gerechtfertigt sein. Grundsätzlich gelten diese Erwägungen auch für Erwachsene, wobei diese Frage kontrovers diskutiert wird und unterschiedliche Praxen bestehen. Die vorliegenden Richtlinien gehen davon aus, dass die Nahrungsund Flüssigkeitszufuhr bei chronisch Kranken, noch nicht Sterbenden, primär der Stabilisierung und dem Erhalt der körperlichen Integrität (z.B. Dekubitus-Prophylaxe) dient.

\section{Empfehlungen zuhanden der zuständigen Gesundheitsbehörden}

Angesichts der Fortschritte bei lebenserhaltenden medizinischen Massnahmen und der hohen Anforderungen an die Pflege der Betroffenen können die vorhandenen Ressourcen an Grenzen stossen. Die Verantwortlichen des Gesundheitswesens sollten mit ihrer Politik gewährleisten, dass alle diese Patienten ohne ökonomische Rücksichten im Sinne der RL behandelt werden können. Langzeitpatienten mit schwerster Hirnschädigung haben das Recht auf Betreuung, quantitative und qualitative Lebenserhaltung und Linderung ihres Leidens. 